\title{
Erratum: Prospects for atomic clocks based on large ion crystals [Phys. Rev. A 92, 032108 (2015)]
}

Kyle Arnold, Elnur Hajiyev, Eduardo Paez, Chern Hui Lee, M. D. Barrett, and John Bollinger (Received 3 April 2017; published 17 April 2017)

DOI: 10.1103/PhysRevA.95.049904

In our discussion of higher-order micromotion effects, we neglected contributions from higher Fourier components in Eq. (14) and mistakenly evaluated a dc term to balance the pseudopotential in Eq. (18). Since the only force that acts on an ion is the electric field that causes the Stark shift, the shift is proportional to $\langle\ddot{r}\rangle$ and hence the dc term does not contribute. This changes Eq. (17) to

$$
\begin{aligned}
\frac{\Delta v_{i}}{v}= & -\left(\frac{\omega_{z} l}{2 c}\right)^{2}\left[1-\left(\frac{\Omega}{\Omega_{0}}\right)^{2}\right]\left\{\mathbf{R}_{0, i}^{T} \Lambda_{\mathrm{rf}}^{2} \mathbf{R}_{0, i}-\frac{\epsilon^{2}}{2} \mathbf{R}_{0, i}^{T} \Lambda_{\mathrm{rf}} \mathbf{W}_{0, i}+\frac{\epsilon^{2}}{2} \mathbf{R}_{0, i}^{T} \Lambda_{\mathrm{rf}}\left(\Lambda_{\mathrm{s}}+\frac{1}{16} \Lambda_{\mathrm{rf}}^{2}\right) \Lambda_{\mathrm{rf}} \mathbf{R}_{0, i}+\frac{\epsilon^{2}}{16} \mathbf{R}_{0, i}^{T} \Lambda_{\mathrm{rf}}^{4} \mathbf{R}_{0, i}\right\} \\
& +\left(\frac{\omega_{z} l}{2 c}\right)^{2} \frac{3 \epsilon^{2}}{64} \mathbf{R}_{0, i}^{T} \Lambda_{\mathrm{rf}}^{4} \mathbf{R}_{0, i}
\end{aligned}
$$

and eliminates the need for Eqs. (18) and (19). Consequently Eq. (22) becomes

$$
\frac{\Delta v_{i}}{v}=-\left(\frac{a \omega_{z} l}{2 c}\right)^{2}\left\{\left[\lambda_{0}-\left(\frac{\Omega}{\Omega_{0}}\right)^{2}\right] \lambda_{1} \mathbf{R}_{0, i}^{T} \Lambda^{2} \mathbf{R}_{0, i}-\frac{\epsilon^{2}}{2 a}\left[1-\left(\frac{\Omega}{\Omega_{0}}\right)^{2}\right] \mathbf{R}_{0, i}^{T} \Lambda \mathbf{W}_{0, i}+\frac{\epsilon^{2} \delta}{2}\left[1-\left(\frac{\Omega}{\Omega_{0}}\right)^{2}\right] \mathbf{R}_{0, i}^{T} \Lambda \mathbf{R}_{0, i}\right\},
$$

with Eq. (23) changed to

$$
\lambda_{0}=1-\frac{3 a^{2}}{64} \frac{\epsilon^{2}}{\lambda_{1}} \text { and } \quad \lambda_{1}=1+\frac{3 a^{2}-8}{32} \epsilon^{2} .
$$

This diminishes the effect of transverse asymmetry, $\delta$.

For the symmetric case, Eq. (25) does not change but the constants in Eq. (26) are changed to

$$
\lambda_{0}^{\prime}=1-\frac{9}{64} \frac{\epsilon^{2}}{\lambda_{1}^{\prime}} \quad \text { and } \quad \lambda_{1}^{\prime}=1+\frac{21}{160} \epsilon^{2}
$$

These changes do not affect the general discussions or conclusions of the paper.

We thank Georgy Kazakov, who is preparing a manuscript that will include an independent calculation, for bringing this to our attention. 